\title{
Rackham: An Interactive Robot-Guide
}

\author{
Aurélie Clodic*, Sara Fleury*, Rachid Alami*, Raja Chatila*, Gérard Bailly ${ }^{\ddagger}$, Ludovic Brèthes*, \\ Maxime Cottret*, Patrick Danès*, Xavier Dollat*, Frédéric Elisei ${ }^{\ddagger}$, Isabelle Ferrané ${ }^{*}$, Matthieu Herrb* \\ Guillaume Infantes*, Christian Lemaire*, Frédéric Lerasle*, Jérôme Manhes*, Patrick Marcoul*, \\ Paulo Menezes*, Vincent Montreuil*
}

\author{
* LAAS - CNRS \\ 7, Avenue du Colonel Roche \\ 31077 Toulouse, France \\ Email: firstname.lastname@laas.fr
}

\author{
$\dagger$ IRIT, Université Paul Sabatier, \\ 118 Route de Narbonne \\ 31062 Toulouse, France \\ Email: firstname.lastname@irit.fr
}

\author{
¥Institut de la Communication Parlée \\ 46 Avenue Félix Viallet \\ 38031 Grenoble, France \\ Email: lastname@icp.inpg.fr
}

\begin{abstract}
Rackham is an interactive robot-guide that has been used in several places and exhibitions. This paper presents its design and reports on results that have been obtained after its deployment in a permanent exhibition. The project is conducted so as to incrementally enhance the robot functional and decisional capabilities based on the observation of the interaction between the public and the robot.

Besides robustness and efficiency in the robot navigation abilities in a dynamic environment, our focus was to develop and test a methodology to integrate human-robot interaction abilities in a systematic way.

We first present the robot and some of its key design issues. Then, we discuss a number of lessons that we have drawn from its use in interaction with the public and how that will serve to refine our design choices and to enhance robot efficiency and acceptability.
\end{abstract}

\section{INTRODUCTION}

In recent years, several autonomous robots, developed by research labs and universities, have been deployed in public areas. Taking robots out of the laboratories is a difficult challenge as they face new environments where they have to act autonomously among a large public not aware of their capacities or limitations. In order to be interesting and accepted they have to attract the attention of the public and to adopt understandable behaviors. They have of course to be robust to a large class of situations and to act safely for the public and for themselves. Besides, there is a crucial need of flexibility, modularity and programmability for such systems.

Rhino[5] and Minerva[20] have been the precursors of a series of tour-guide robots in various museums and exhibition halls [15] and culminated at the Swiss National Exhibition Expo 02 where 11 robots were demonstrated for 159 days [19].

These tour-guide robots had various degrees of autonomy and were using more or less sophisticated techniques for navigation.

However, they have all pointed out that studying humanrobot interaction was necessary, in its definition as well as its

The work described in this paper was conducted within the EU Integrated Project COGNIRON ("The Cognitive Companion") and was funded by the European Commission Division FP6-IST Future and Emerging Technologies under Contract FP6-002020 and by the French National Program ROBEA. implementation. It appeared that robots must obey to some "social" clues [8] and led to the development of service robots (e.g. Pearl [17], Care-O-bot II [9], CERO [10], Lino [12] and BIRON [22]).

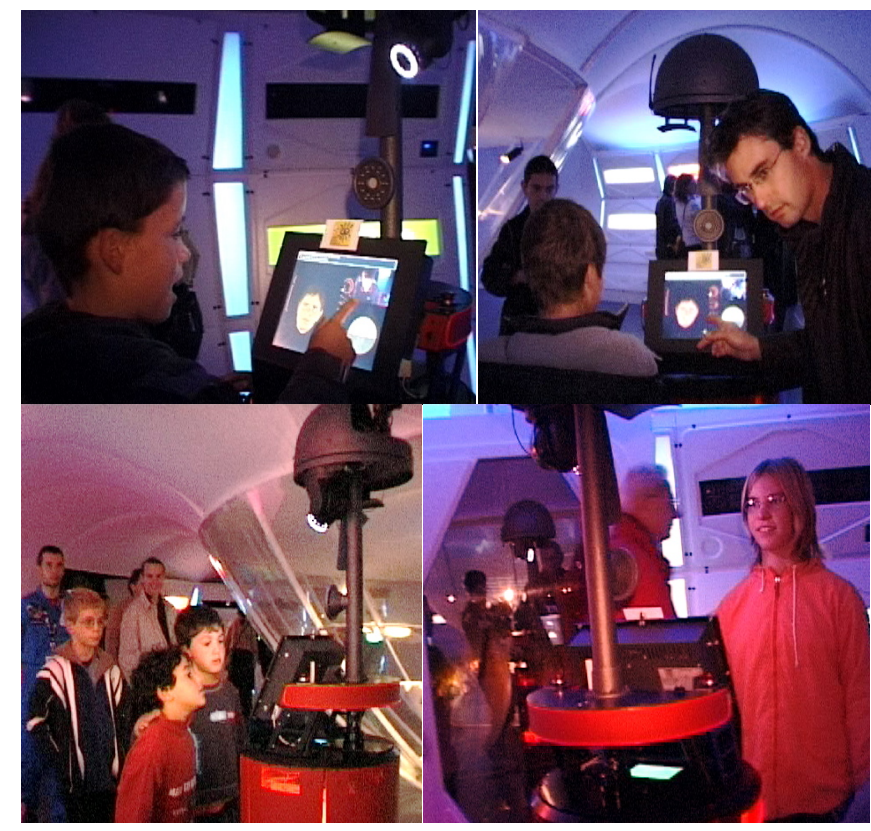

Fig. 1. Some Rackham's fans.

To study human-robot interaction realistically, an experimentation environment must be found, out of a laboratory and its standard rooms and halls... and its robotics scientists who know very well how their "creatures" work. We have deployed our robot, named Rackham, on its own for periods of 2 weeks every 3 months in an exhibition at a science museum in Toulouse.

In this paper, we describe this tour-guide robot. We begin with a presentation of the exhibition context. Next, we describe the LAAS software architecture ([1]) and the various tools already developed to implement Rackham functionalities. Then, we present the robot supervisor, which a part of the architecture and describe its operation. We conclude with 
experimental results, comments and analysis.

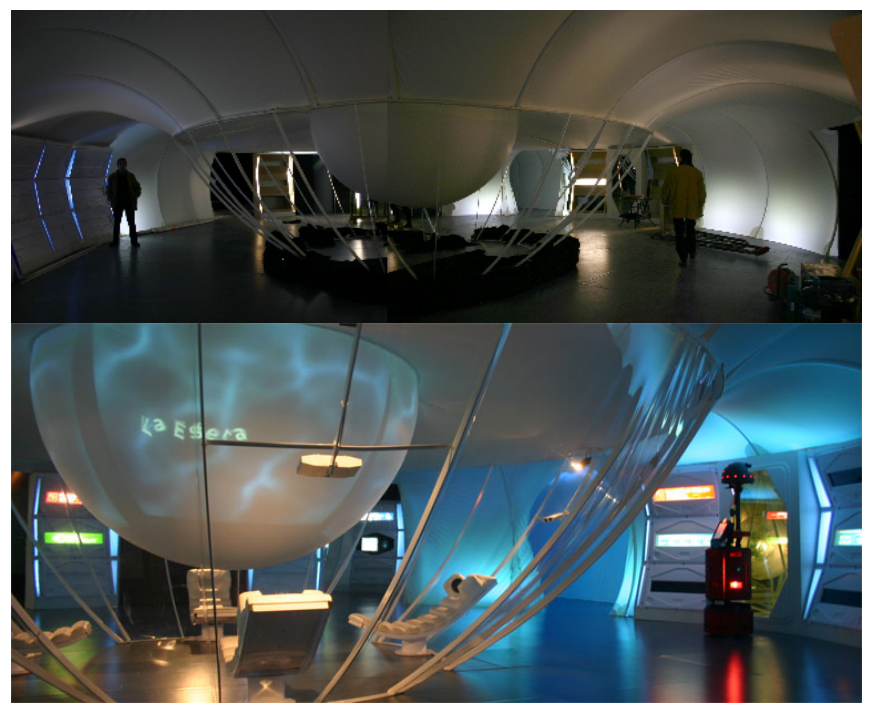

Fig. 2. The Tsiolkovski spaceship: a difficult environment for navigation.

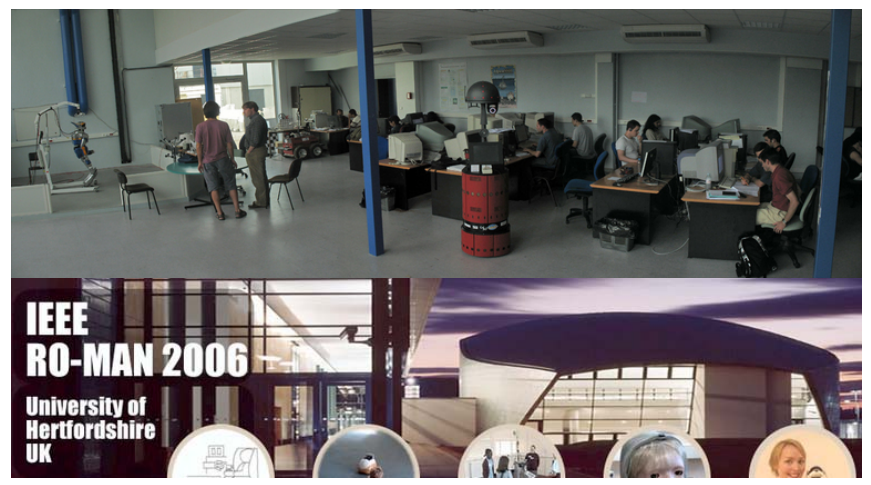

Fig. 3. Three (very) different environments and navigation contexts: the Cité de l'Espace (figure 2), the LAAS robotics laboratory, and RO-MAN 06 at Hertfordshire ... still to be descovered.

\section{The EXPERIMEnTAl CONTEXT AND SCEnARII}

\section{A. The Robot Rackham}

Rackham is a B21r robot from iRobot. It is a 4-feet (118 $\mathrm{cm})$ tall and 20-inches $(52 \mathrm{~cm})$ wide cylinder topped with a mast supporting a kind of helmet. It integrates 2 PCs (one mono-CPU and one bi-CPUs running P3 at $1 \mathrm{GHz}$ ). We have extended the standard equipment with a pan-tilt Sony camera EVI-D70 attached under the helmet, a digital camera mounted on a Directed Perception pan-tilt unit, a ELO touch screen, a pair of loudspeakers, a microphone, an optical fiber gyroscope and wireless Ethernet.

In order to integrate all these components in a robust and pleasant way the "Cité de l'Espace" has designed a "head" on a mast, toped by a helmet which represents a kind of oneeyed modern pirate or an African art statue (see Figure 4). The eye is materialized by the EVI-D70 camera fixed upsidedown above the helmet, the second camera is hidden in the helmet and one loudspeaker is integrated in the "mouth".
The "nose" is only decorative. The mast has been designed as high as possible to keep the cameras away from children's hands.

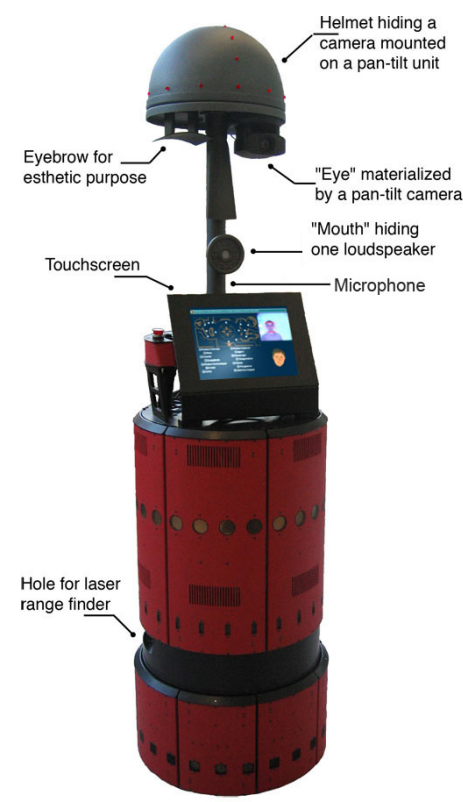

Fig. 4. Rackham and its equipment.

\section{B. Mission Biospace}

Mission BioSpace is an exhibition designed by the "Cité de l'Espace" to illustrate what could be an inhabited spaceship by the way of fourteen interactive elements that propose a vision of the future. Rackham "lives" in the spaceship and proposes to guide visitors to the different exhibits, leading them to the interest spot of their choice and introducing the different exhibits to them.

\section{A difficult context for navigation and interactions}

The exhibition simulates the interiors of an imaginary 25x10 square meters spaceship called Tsiolkovski, including visual and acoustic atmosphere. Hence it represents a difficult context for navigation and interaction (see Figure 2):

- ambient noise makes speech synthesis and voice recognition difficult to understand,

- the room is dark with changing background colors,

- supple walls made of tight cloth difficult to model,

- prominent obstacles on the ground and at head height, not visible by the robot proximity sensors,

- some translucent obstacles not perceived by the laser range finder,

- some narrow passages, which require a precise positioning of the robot to navigate through them,

- crowded environment.

${ }^{1}$ The "Cité de l'Espace" is a space adventure park (http://www.citeespace.com) located in Toulouse, France. 


\section{A typical Rackham mission}

When Rackham is left alone with no mission, it tries to find people to interact with. As soon as a person is detected, thanks to visual face detection, Rackham introduces itself through a virtual 3D face on its touch screen "I'm Rackham and I can guide you in this spaceship" or alternatively it explains how to use its services: "Ask for a destination using the microphone or the touch screen".

If the visitor selects a destination Rackham first confirms its new mission "OK, I will guide you to...", then plans and displays its trajectory and invites the visitor to follow it.

While navigating, the robot keeps on giving information about the progress of the ongoing journey: a congestion will require to temporarily stop or even to compute an alternative trajectory while a too important uncertainty on the position might call for a relocalisation procedure; temporary disappearance and reappearance of the guided visitor from the field of view is also detected and indicated by sentences such as "Where are you?", "Here you are again!". Using various buttons displayed on the interface, the visitor may stop and change the ongoing mission.

\section{Robot Software Architecture}

\section{A. Overview}

The software architecture is an instance of the LAAS $^{2}$ architecture [1]. This instance includes two layers of the architecture including a supervisor written with openPRS ${ }^{3}$ (a Procedural Reasoning System) that controls a distributed set of functional modules.

A module is an independent software component that can integrate a set of functions with various time constraints or algorithmic complexity: control of sensors and actuators, servo-controls, monitoring, data processing, trajectory computation, etc.

Each module is created using a module generator tool called GenoM and thus presents standard behavior and interfaces [7]. The functions encapsulated in a module can be dynamically started, interrupted or (re)parameterized upon asynchronous standard requests sent by the supervisor.

Once started, a service runs autonomously. A final reply that qualifies how the service has been executed is returned to the supervisor with the end of the service. During the execution a module can export data in structured public entities called posters and read data from posters produced by other modules (eg, robot positions, trajectories, maps and so on). The set of posters represent a distributed database of the state of the functional level of the architecture.

For Rackham, we have implemented 16 modules. We now present them according to their role in the system (see Figure 5). All the modules are controlled by the supervisor according to the ongoing mission, the context and the robot state.

\footnotetext{
2LAAS stands for: "LAAS Architecture for Autonomous Systems".

${ }^{3}$ The set of tools used to build an instance of this architecture (GenoM, openPRS, pocolibs, etc) are freely distributed at: http://softs.laas.fr/openrobots.
}

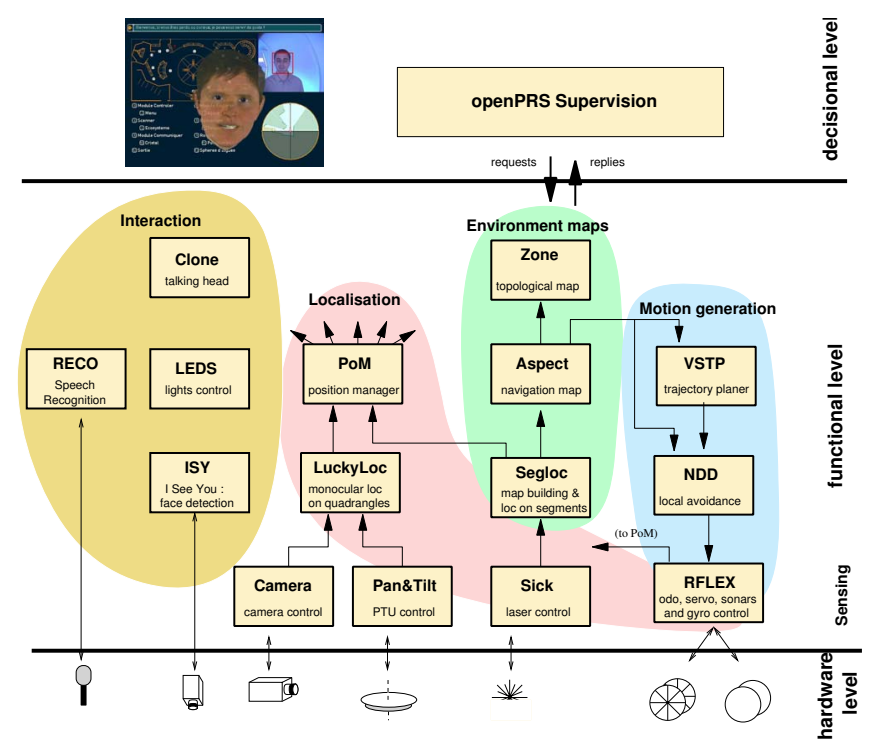

Fig. 5. The functional level of Rackham and its 16 modules.

\section{B. Localization}

Several modules are involved in the localization of the robot. First the rflex module, which interfaces low level software provided by the manufacturer and which exports in a poster the position computed by the odometry and corrected by the gyroscope. This position provides a good estimate of the motions of the robot. It is associated with a covariance matrix deduced from a probabilistic model error.

To localize itself within its environment the robot uses a SICK laser, controlled by the sick module, that exports at the required rate the laser echoes together with segments deduced from aligned echoes. Another module, segloc, matches these segments with segments previously recorded in a map thanks to a classical SLAM procedure. However the map (see Figure 6) is updated only during exhibition closing time (no public).

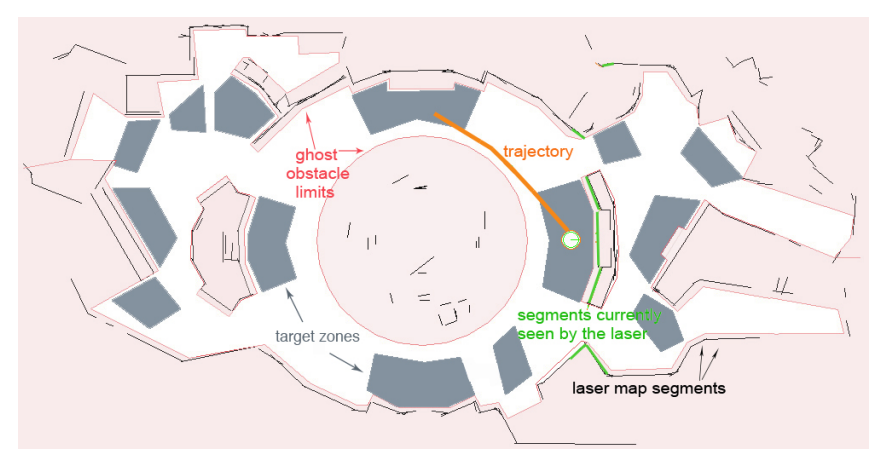

Fig. 6. The map of the environment built by Rackham contains 232 segments (black). It has been augmented with virtual obstacles (green or dark-grey) and target zones (light-gray).

The localization being a very critical ability, a third localization modality, based on vision, has been designed. The camera is controlled by the module camera that produces images to be processed by another module called luckyloc 
that extracts, identifies and localizes planar quadrangles that appear on the furniture. However, we never had to use this redundant function.

Finally, the various uncertain positions exported by the modules rflex, segloc and luckyloc are merged by pom, the position manager module. This module is able to integrate positions computed at various frequencies and even to propagate "old" position data. Various fusion strategies can be selected like Kalman fusion or integration of the measured motions relatively to the most reliable positions. The supervisor is informed in case of localization problems with one of the modules, fusion difficulties or significant uncertainties on the position. Depending on the problem and the context, various strategies are applied.

It is important to note that the pom module allows to centralize the robot positions and to export one and only one reference position. All the other system components do not need to know how this position is obtained. This procedure can change dynamically without disturbing the position consumers. It is a very important mechanism to manage redundancy and an essential feature for this critical function.

On top of this geometric positioning, several topological zones corresponding to places of special interest ("TARGETS"), to dangers for the navigation ("OBSTACLES") not always visible by the robot sensors like prominent or transparent furniture), or to other special areas ("SPECIAL") have been defined in the environment. The zone module continuously monitors entrance and exit of the robot from these zones and informs the supervisor.

\section{Obstacle and people detection}

Obstacle detection is a critical function both for security reasons and for interaction purposes. The most efficient sensor is once again the laser. However Rackham's laser can only look forward (over 180 degrees) in an horizontal plane. To partially overcome these limitations, the laser data are integrated in a local map by the aspect module and filtered using knowledge about the global map (segments and the virtual obstacles ${ }^{4}$ ). Thus, aspect exports, every $40 \mathrm{~ms}$, a local map of the surroundings of the robot which represents the free space and which distinguishes static (ie, that belong to the environment or the virtual obstacles) and dynamic obstacles (probably visitors). This local map is permanently displayed on the bottom right of the interface (see Figure 7). Using this representation, aspect is able to inform the supervisor when the robot is surrounded by unpredicted obstacles. The red leds on the helmet flicker at a frequency proportional to the obstruction density of dynamic obstacles.

A much more robust people detector is offered by the module called isy (or, "I See You") which is able to detect faces in real time from a color camera image. The detector uses a cascaded classifier and a head tracker based on a

${ }^{4}$ Let us recall that in the context of Mission BioSpace with prominent and transparent obstacles this notion of virtual obstacles is very important. particle filter [4]. Isy controls the camera orientation in order to track the detected face. It informs the supervisor when it detects or looses a face. From the direction and the size of the face it is able to estimate the $3 \mathrm{D}$ position of the detected person with a sufficient accuracy (about $10 \mathrm{~cm}$ for the height and $20 \mathrm{~cm}$ for the range).

The ambient light (weak and changing) of the space ship does not provide enough light; a ring of white leds fixed around the lens provide a range of detection of about three meters.

\section{Trajectory and motion}

Rackham being a guide, it must be able to take visitors to various places in the exhibition and that are displayed on the interactive map. For the robot they correspond to polygonal target zones (see §III-B) and to the position of the associated element of interest (which can be itself out of the polygon) that the robot will have to comment.

Robot motion involves mainly three modules:

- rflex that manages the lower servo-control loop, transmitting the reference speeds at the micro-controller.

- ndd integrates a local avoidance procedure based on an algebraic instance of Nearness Diagrams [14]. The input obstacles are provided by the aspect map (see $\S$ III-C).

- vstp is a Very Simple [but very efficient] Trajectory Planner based on an algebraic visibility graph optimized with hash tables ${ }^{5}$. A main visibility graph is precomputed for the static segments of the map. Dynamic obstacles can be added and removed in real-time upon supervisor requests.

The strategy used to coordinate the implied modules is dynamically established by the supervisor. The objective is of course to reach the target zone while avoiding obstacles. The planned trajectory is an Ariadne's thread for ndd: the vertices of the broken line are sub-goals. Usually the supervisor has to intervene only if ndd does not make progress towards the goal. In such a case, various strategies can be applied: computing of a new trajectory taking into account the encountered obstacles, waiting for a while, starting an interaction with people around, etc. The motion is over when the robot is inside the target zone.The maximum speed that the robot can achieve in this mode is about 0.6 meters per second, but of course the speed is dynamically adapted according to the distance and the position of surrounding obstacles.

\section{E. Interactions}

For now, the interactions are mainly established through the following components:

- the dynamic "obstacle" detector aspect,

- the isy face detector,

- the voice recognition,

- the displays and inputs from the touch screen,

- the speech synthesis with the 3D animated face,

- the control of the robots' lights.

${ }^{5}$ VSTP is freely distributed: http://softs.laas.fr/openrobots/. 
While the first ones allow to detect the presence or the departure of people, the last ones permit the robot to "express" itself and thus establish exchanges.

The vocal synthesis is highly enriched by a 3D animated head displayed on the screen. This talking head, or clone, is developed by the Institut de la Communication Parlée (http://www.icp.inpg.fr). The clone is based on a very accurate articulatory 3D model of the postures of a speaking locutor with realistic synthetic rendering thanks to 3D texture projection. From a given text, the speech synthesizer produces coordinated voice and facial movements (jaw, teeth, lips, etc.).

The directions of the head and of the eyes can be dynamically controlled. This capability is important as it allows to reinforce an interaction, looking towards the interlocutor face detected by isy, or to point out an object or a part of the exhibition currently mentioned by the robot.

The clone appears in front of the touch-screen each time the robot has to speak (see Figure 7). Meaningful messages have been prepared, corresponding to the various situations encountered by the robot or to the places that need to be described during the visit.

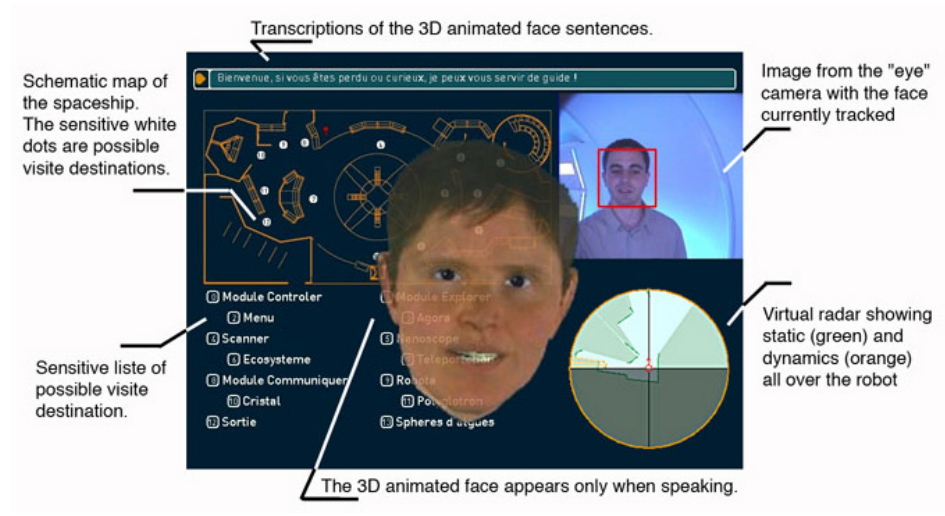

Fig. 7. A view of the interface of the touch-screen.

A French speech recognition system has been developed by IRIT laboratory in order to allow visitors to interact with the robot, by means of some basic vocal commands. Based on the grammar-based open source recognition engine, Julian (http://julius.sourceforge.jp/en/julius.html), this automatic speech recognition system is intended to recognize two types of vocal commands: (1) basic control and movement commands and (2) guidance commands towards one of available destinations.

Three specific linguistic resources are necessary for the recognition process: acoustic models (phones), a word lexicon including word pronunciations, and a grammar describing the possible syntax of word sequences on the specific task. A French word lexicon (150 words / 250 pronunciation variants) and a specific grammar (300 rules) have been specially designed for Rackham and its dedicated tasks at the Cité de l'Espace. It has been eleborated from hundreds of recordings taken with help of wizard of $\mathrm{Oz}$ experiments (the sound was initially transmitted to an operator - thanks to a direct connection of the microphone on a walky-talky which in his turn sent the adapted command to the onboardsupervisor via a wifi connection to the robot). Then, the utterances were manually transcripted, and the word lexicon was set up with the French usual words encountered in these transcriptions as well as all the location names specifically related to the City of Space and the Biospace Mission. Word pronunciations were mainly obtained from BDLEX and its lexical and phonological resources, which are available at IRIT. The acoustic models were directly taken from previous works carried out in the Samova team (IRIT). They were designed to be speaker independant and were used without any adaptation to this new acoustic environment, to this specific task or to any new speaker.

Then, once a speaker utterance is recorded, the engine searches for the most likely word sequence according to the constraints given by the grammar. Then the best sentence is converted into a specific command processed by the supervisor. Despite the very difficult sound conditions and the great diversity of visitor's voices, the recognition performance was of $84 \%$. The visitors always kept the possibility to interact through the touch screen. But about $60 \%$ still used the voice recognition instead of the touch screen.

The robot interface, written in Java, is made of independent components or microGUIs directly controlled by the supervisor through a dedicated communication channel.

The available microguis are (Figure 7):

- a map of the environment including the current robot position and trajectory,

- the local "aspect" map displayed as a radar,

- the image of the "eye" camera with the faces currently detected by isy,

- the clone or talking head,

- pop-up warning messages,

- top messages,

- localization window (init).

This set of modules offers a good degree of redundancy for several functions as shown on Figure 5. It greatly helps in making the robot's behavior robust, and provide tools for the supervisor to adapt to a large set of varying situations. The supervisor is briefly described in the next section.

\section{SUPERVISION}

\section{A. General Description}

We have developed an agent-based supervision system that deals with tasks in terms of individual tasks (only the robot is involved), joint tasks (in which the robot and a person are involved) and activities that correspond to low level actions that are not further decomposed.

Joint tasks are based on Joint Intention Theory (JIT)[6]. In JIT, mutual belief represents a belief that is shared by two agents, i.e. one agent has a belief about something AND has a belief about the other agent beliefs (and vice versa as illustrated in figure 8(left).

In our case, we would like the robot not only to act but also maintain a "shared mental state" between itself 


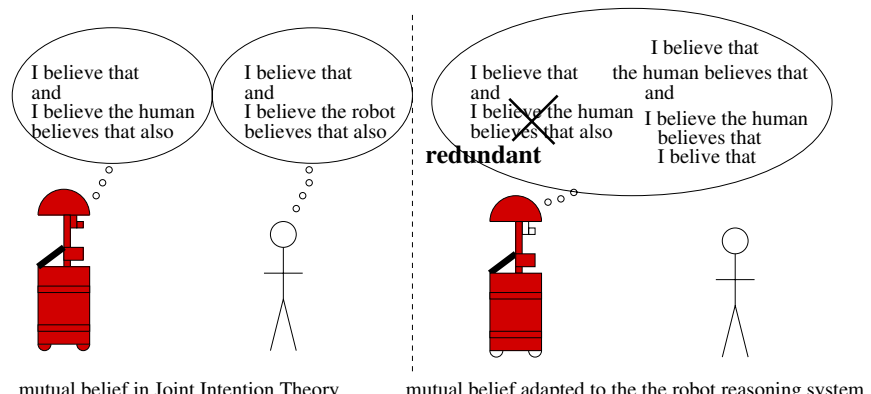

Fig. 8. Joint intention theory transposed to the robot reasoning system, the robot takes into account the human perspective

and its human partner. It makes observations and deductions concerning the beliefs and commitments of the human (and this is inherently a partially observable information from the robot point of view...) and has to behave in a way that is easily understood by its human partner (figure 8-right). Our model includes three perspectives which are complementary: the personal robot perspective, the robot's belief about the human personal perspective, and finally the robot's belief about the human belief's concerning itself. This has to do with perspective taking, "the ability of a person to take someone else's perspective" [16].

Also related to JIT is the notion of commitment and task state that are managed by the robot supervisor. We define:

(COMMITMENT \$task \$who1 \$who2 \$value) $\$$ value $=$ YES or NO

(STATE \$task \$who1 \$who2 \$value) $\$$ value $=$ UNACHIEVED or ACHIEVED or IRRELEVANT or IMPOSSIBLE

that has to be read as robot's belief about \$whol's belief concerning \$who2 state or commitment to the \$task.

Each task is refined as a plan and dedicated monitors. A plan corresponds to a succession of sub-tasks and/or activities. Monitors serve to assess commitment and task state variables, each of these monitor can be seen as many as focus of attention [21] on a particular point at a given level.

Consequently, the system can be controlled at different levels at the same time. If something is detected at a given level, the system is able to take it into account at that level by applying relevant solutions and propagating events, when necessary, towards the higher or the lower levels.

Monitors are implemented in order to comply with multiple modalities. Indeed, in HRI, it is often the case that a given information comes through various ways (e.g. a human can give the same order by gesture, by speech, by the help of a touchscreen or by a combination of these modalities).

In the current implementation, the supervisor is written in Open-PRS ([11], see "http://softs.laas.fr/openrobots/").

\section{B. An example of Rackham missions}

After the Initialisation task, Rackham is left alone in the exhibition. With nobody in its vicinity, Rackham launches an individual task called Looking-for a-visitor. During this task, Rackham roams in the exhibition room in search of visitors: (STATE Looking-for-a-visitor Rackham Rackham unachieved) and (COMMITMENT Looking-for-a-visitor Rackham Rackham YES).

It has three ways to detect them: isy face detection, the speech recognition and the touchscreen. When a person is detected, a new agent is created and the task Looking-for-avisitor is ended. Rackham will now try to propose the joint task Guide to the person we will become the visitor.

The first thing to obtain concerning a joint task is commitment from both parts, to do that we will study three variables:

- Rackham commitment to the task: (COMMITMENT Guide Rackham Rackham \$value)

- Rackham's belief concerning the visitor commitment to the task: (COMMITMENT Guide Visitor Visitor \$value),

- Rackham's belief concerning the visitor's belief about Rackham commitment to the task:

(COMMITMENT Guide Visitor Rackham \$value).

The robot is committed to the task so (COMMITMENT Guide Rackham Rackham YES). Now, Rackham will inform the visitor that it is committed to the task and propose him to participate by the help of the task Propose-toGuide, this task should be seen as a request procedure in the sense of communicative acts [13]. Now, we have also: (COMMITMENT Guide Visitor Rackham YES).

Then, either the visitor answers directly by choosing a destination and we will have (COMMITMENT Guide Visitor Rackham YES), or he will not and Rackham will employ an Ask-Agreement task.

After that, we will have (COMMITMENT Guide Visitor Rackham YES) or (COMMITMENT Guide Visitor Rackham NO), if e.g. the visitor goes away. We will now consider the first case.

So, commitment to the task is established by both parts. Then the robot confirms "ok, i will guide you to that destination. Please, follow me.". The task achievement begins. Rackham will bring the visitor to the destination and the visitor is supposed to follow it and everyone is supposed to consider the task as unachieved (see figure 9).

We will now study tasks and sub-tasks progress state. For instance, in the case the visitor goes away, Rackham will react first by stopping; then it will ask and display "where are you ?". The state will change to UNACHIEVED and the task will be re-started if the visitor comes back (detected by the help of the face detection or if he touches the screen). If he does not, the state will change to IMPOSSIBLE and the task will be abandoned.

At the end of the task, Rackham also has to ensure that there is a mutual belief concerning the achievement of the task.

We have used this supervision system on Rackham during its last stays at the Space City Museum in Toulouse; 1/4 of the time was spent in Looking-for-a- visitor and 3/4 of the time, Rackham performed the Guide joint task. 


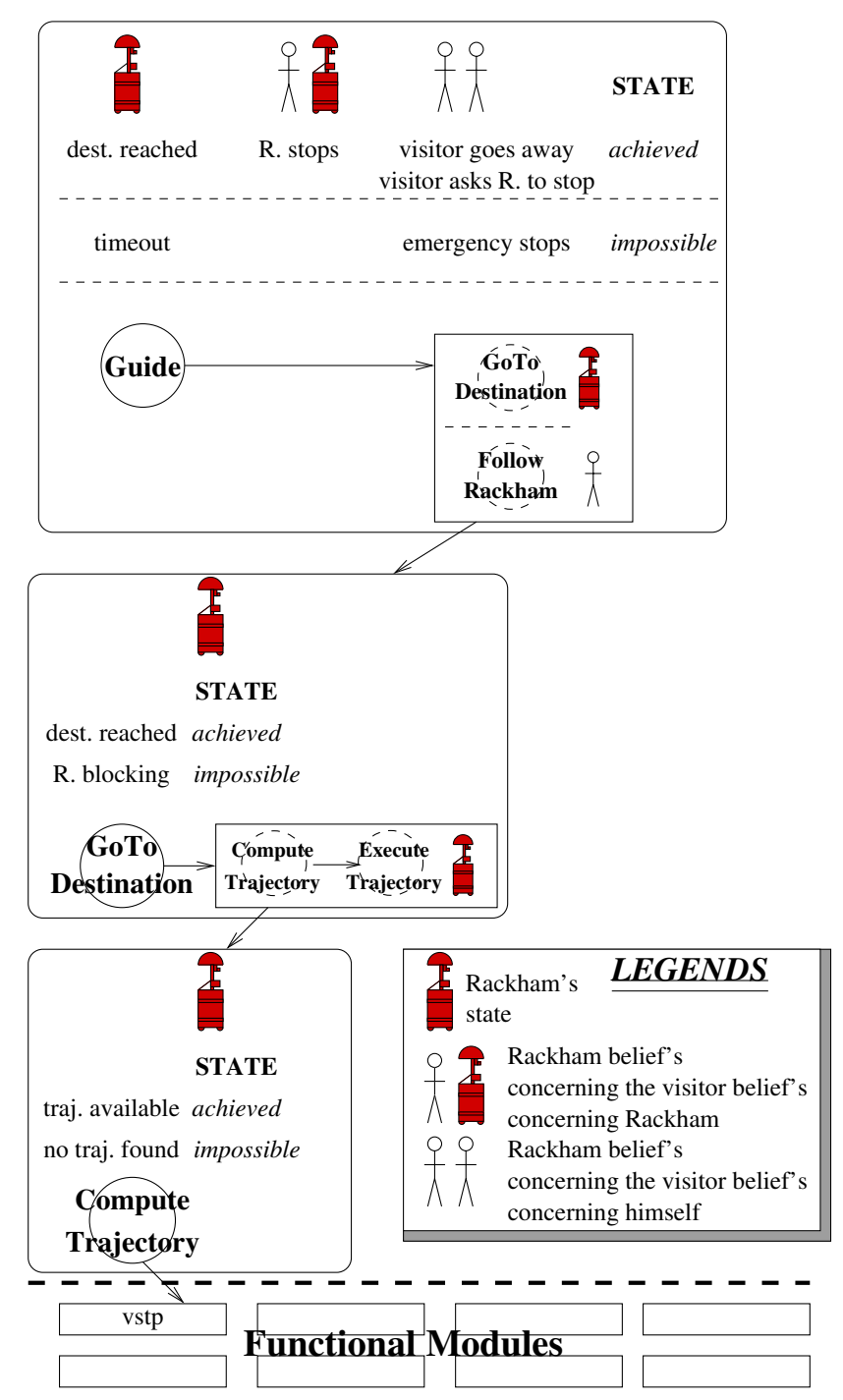

Fig. 9. Tasks hierarchy : This figure illustrates a snapshot of the incremental task refinement hierarchy and the associated monitors.

\section{RESULTS AND ANALYSIS}

\section{A. Quantitative results}

Between March 2004 and November 2006, Rackham has "worked" about 100 days at the "Cité de l'Espace" in nine venues ${ }^{6}$ (12 effective days per venue).

During these venues it was in mission for 200 hours, carrying out about 6000 missions over $66 \mathrm{~km}$ on the solicitation of 3000 visitors.

The robot was sufficiently robust to be operated by the personnel of the Cité de l'Espace without our intervention. It was very well accepted by the public.

We collected various data for analysis purposes: all the requests to the modules and their reply, the covered distance, the visitors interactions, etc.

The results presented below are a synthesis of the data collected during the period from February 7th to February 20th. During this stay Rackham was put on mission by

\footnotetext{
${ }^{6}$ See http://www.laas.fr/robots/rackham.
}

the organizers for a total of 26 hours. The robot is then permanently in interaction and was requested for 832 visits to a place of interest. About $20 \%$ of these missions were voluntarily interrupted by the visitors before the end. For about $2 \%$ of the missions the supervisor has detected a problem (mainly an abnormal delay to refresh a low level data and few logical inconsistencies) and called for the operator for security reasons.

The total covered distance is about $9 \mathrm{~km}$ (for an environment of $25 \times 10$ square meters). The average speed of about $0.54 \mathrm{~km} / \mathrm{h}$ integrates all the disturbances (crowds, immobile and dense group of visitors) during the motion.

\begin{tabular}{|c|c|c|c|c|c|}
\hline \multicolumn{6}{|c|}{ From February 7, 2005 to February 20,2005} \\
\hline day & $\begin{array}{c}\text { number } \\
\text { of } \\
\text { missions }\end{array}$ & $\begin{array}{l}\text { distance } \\
\text { in } \\
\text { meters }\end{array}$ & $\begin{array}{l}\text { duration } \\
\text { hh:mn } \\
\text { (motion) }\end{array}$ & $\begin{array}{l}\text { number } \\
\text { of } \\
\text { requests }\end{array}$ & $\begin{array}{c}\text { average } \\
\text { speed } \\
(\mathrm{km} / \mathrm{h})\end{array}$ \\
\hline 1 & 40 & 395 & $1: 25$ & 2801 & 0.51 \\
\hline 2 & 49 & 555 & $1: 32$ & 2719 & 0.56 \\
\hline 3 & 44 & 487 & $1: 18$ & 2557 & 0.62 \\
\hline 4 & 82 & 851 & $3: 32$ & 4338 & 0.44 \\
\hline 5 & 82 & 881 & $2: 28$ & 4209 & 0.58 \\
\hline 6 & 70 & 739 & $1: 49$ & 3609 & 0.56 \\
\hline 7 & 85 & 884 & $2: 14$ & 4338 & 0.50 \\
\hline 8 & 71 & 815 & $2: 24$ & 3984 & 0.53 \\
\hline 9 & 55 & 663 & $1: 31$ & 3154 & 0.60 \\
\hline 10 & 78 & 912 & $2: 29$ & 4742 & 0.49 \\
\hline 11 & 71 & 872 & $2: 08$ & 4214 & 0.54 \\
\hline 12 & 91 & 994 & $2: 49$ & 4632 & 0.53 \\
\hline \multirow[t]{2}{*}{13} & 14 & 161 & $0: 27$ & 733 & \\
\hline & 832 & $9209 \mathrm{~m}$ & 26:06 & 46030 & 0.54 \\
\hline
\end{tabular}

All these figures are only related to on-going missions directly required by a visitor. However, when Rackham was not solicited, it wandered by itself in order to meet visitors. Once a visitor was detected thanks to the eyecamera, Rackham offered vocally its services and presented the touch-screen. This behavior ran about once over two missions and occupied Rackham 30\% more time moving around. In $70 \%$ of these "huntings for visitors", Rackham succeeded in its quest and found a "face" to interact with.

\section{B. Analysis}

The figures of all these experiments can be considered satisfactory. Moreover, it is important to note that the robot never collided visitors nor furniture! For such exhibitions, the robustness of the low-level autonomous navigation capacities is fundamental. The coherence, the plausibility and the "freshness" of the data (laser, references, positions, etc.) were continuously checked. Most of the "failures" detected by the supervisor were false alarms easily deactived later on.

From a more quantitative viewpoint, we were very happy to see that Rackham was immediately identified as a robot and very well accepted by the public.

However, the quality of the interaction is more difficult to quantify. For instance, we have noticed that a continuous interaction all along the mission is fundamental to keep visitors interest. The robot must continuously check the presence of the guided person, show him that it knows where (s)he is (for instance a video return on a screen is very appreciated). The robot must also continuously explain 
what it is doing (e.g., why it has stopped) and what are its intentions (e.g., avoiding visitors to the left).

\section{CONCLUSION AND FUTURE WORK}

In this paper we have proposed an instance of the LAAS architecture that is adapted to human-robot interaction. We have built a supervisor which represents the interaction tasks in addition to traditional navigation tasks and is able to explain its behavior and to interact with the people in the vicinity of the robot during its mission. We have combined this supervisor with a functional level (implementing localization, motion control with collision avoidance and interaction with users) that provide enough flexibility and redundancy to achieve a certain level of robustness in an relatively "hostile" environment.

Rackham now has solid foundations which allow it to navigate in a robust manner and to establish a simple interaction with people in a real world environment. It has been effectively used quite intensively and is considered as an attractive and successful component of the overall exhibition.

But this is only the first page of the story. We now work to enhance Rackham's interaction and perception capabilities. The integration of such capabilities will be done with the concern of developing a systematic manner to integrate more sophisticated context interpretation and to provide decisionmaking to synthesize and control interactive tasks at various levels.
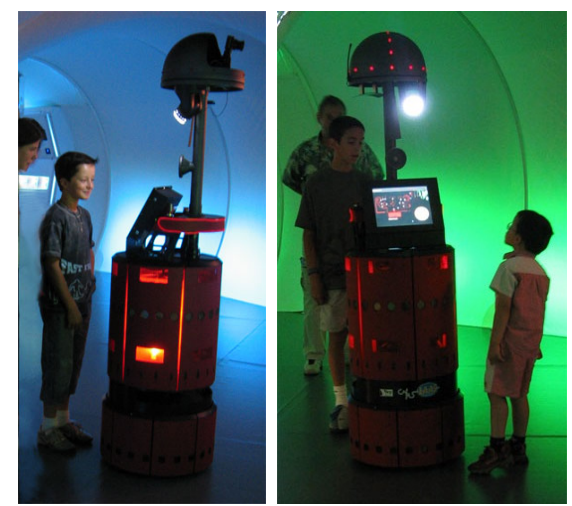

Fig. 10. The Tsiolkovski spaceship: a difficult environment for navigation.

\section{ACKNOWLEDGMENTS}

Numerous persons have contributed to the project : the authors want to thanks: Aymeric Yvernes, Coline LelongPantel, Florent Lanterna, Stéphane Plais, Abdelatif Baba, and from the Space City Museum: Jean-Noël Plachez, Véronique Hallard, Maxime Randisi and Stéphan Lauriol.

\section{REFERENCES}

[1] R. Alami, R. Chatila, S. Fleury, M. Ghallab, F. Ingrand, "An architecture for autonomy", International Journal of Robotic Research, Vol.17, $\mathrm{N}^{\circ} 4$, pp.315-337, Avril 1998.

[2] G. Bailly, M. Bérar, F. Elisei, and M. Odisio, "Audiovisual speech synthesis", International Journal of Speech Technology, 6:331-346, 2003.
[3] G. Bailly, L. Brèthes, R. Chatila, A. Clodic, J. Crowley, P.Danès, F. Eliseï, S. Fleury, M. Herrb, F. Lerasle, P. Menezes, R. Alami, "HR+: Towards an interactive autonomous robot", 3èmes Journées du Programme ROBEA (ROBEA'2005), Montpellier (France), 29-31 Mars 2005.

[4] L. Brèthes, P. Menezes, F. Lerasle, and J. Hayet, "Face tracking and hand gesture recognition for human robot interaction," International Conference on Robotics and Automation, New Orleans, May 27 - June 12004.

[5] W. Burgard and A.B. Cremers and D. Fox and D. Häehnel and G. Lakemeyer and D. Shulz and W. Steiner and S. Thrun, "Experiences with an interactive museum tour-guide robot", Artificial Intelligence , 114 (1999) 3-55.

[6] P. Cohen and H. Levesque. "Intention is choice with commitment.",Artificial Intelligence, 42(2-3):213-361, 1990.

[7] S.Fleury, M.Herrb, R.Chatila, "GenoM: a Tool for the Specification and the Implementation of Operating Modules in a Distributed Robot Architecture", IEEE/RSJ International Conference on Intelligent Robots and Systems (IROS), Grenoble, France, 1997.

[8] T. Fong, I. Nourbakhsh, K. Dautenhahn, "A survey of socially interactive robots," Robotics and Autonomous Systems, Special issue on Socially Interactive Robots, 42(3-4), 2003.

[9] B. Graf, M. Hans, and R. D. Schraft. "Care-O-bot II - Development of a next generation robotic home assistant",Autonomous Robots, 16(2):193-205, 2004.

[10] H. Huttenrauch and K. Severinson Eklundh. "Fetch-and-carry with CERO: Observations from a long-term user study with a service robot",In Proc. IEEE Int. Workshop on Robot-Human Interactive Communication (RO-MAN), pages 158-163. IEEE Press, 2002.

[11] F. Ingrand and R. Chatila and R. Alami and F. Robert. "PRS: A High Level Supervision and Control Language for Autonomous Mobile Robots", Proceedings of International Conference on Robotics and Automation (ICRA), 1996, Minneapolis, USA.

[12] B. J. A. Krose, J. M. Porta, A. J. N. van Breemen, K. Crucq, M. Nuttin, and E. Demeester. "Lino, the user-interface robot",In European Symposium on Ambient Intelligence (EUSAI), pages 264-274, 2003.

[13] S. Kumar, M. Huber, and P. Cohen. "Representing and executing protocols as joint actions." Proceedings of the First International Joint Conf on Autonomous Agents \& Multi-Agent Systems (AAMAS), 2002.

[14] J. Minguez, J. Osuna, L. Montano. "A Divide and Conquer Strategy based on Situations to Achieve Reactive Collision Avoidance in Troublesome Scenarios.", Proceedings of the IEEE International Conf. on Robotics and Automation (ICRA), 2004. New Orleans, USA.

[15] I. Nourbakhsh, J. Bobenage, S. Grange, R. Lutz, R. Meyer and A. Soto, "An Affective Mobile Educator with a Full-time Job," Artificial Intelligence, vol. 114,num. 1-2, pp. 95-124, 1999.

[16] I. Nourbakhsh and T. Fong, "Human-robot teams on the moon: Challenges and plans (extended abstract)," International Conference on Robotics and Automation (ICRA), Workshop on Fielding MultiRobot Systems, 2005.

[17] J. Pineau, M. Montemerlo, M. Pollack, N. Roy, and S. Thrun, "Towards Robotic Assistants in Nursing Homes: Challenges and Results.," Robotics and Autonomous Systems, 42(3-4), 2003.

[18] J. Pineau and S. Thrun. "An integrated approach to hierarchy and abstraction for POMDPs," Technical Report CMU-RI-TR-02-21 , Carnegie Mellon University, 2002.

[19] R. Siegwart, and et al. (2003) "Robox at Expo.02: A Large Scale Installation of Personal Robots." Special issue on Socially Interactive Robots, Robotics and Autonomous Systems , 42 (2003) 203-222.

[20] S. Thrun and M. Beetz and M. Bennewitz and W. Burgard and A.B. Cremers and F. Dellaert and D. Fox and D. Haehnel and C. Rosenberg and N. Roy and J. Schulte and D. Schulz "Probabilistic Algorithms and the Interactive Museum Tour-Guide Robot Minerva", Journal of Robotics Research, vol. 19, num. 11, 2000.

[21] J. Trafton, N. Cassimatis, M. Bugasjska, D. Prock, F. Mintz, and A. Schultz. "Enabling effective human-robot interaction using perspective-taking in robots." IEEE Transactions on Systems, Man and Cybernetics, 35(4):460-470, 2005.

[22] B. Wrede, A. Haasch, N. Hofemann, S. Hohenner, S. Hüwel, M. Kleinehagenbrock, S. Lang, S. Li, I. Toptsis, G. A. Fink, J. Fritsch, and G. Sagerer. "Research issues for designing robot companions: BIRON as a case study", In P. Drews, editor, Proc. IEEE Conf. on Mechatronics and Robotics, volume 4, pages 1491-1496, Aachen, Germany, September 2004. Eysoldt-Verlag, Aachen. 